\title{
Article
}

\section{NOTCH3 rs1043996 Polymorphism Is Associated with the Occurrence of Alcoholic Liver Cirrhosis Independently of PNPLA3 and TM6SF2 Polymorphisms}

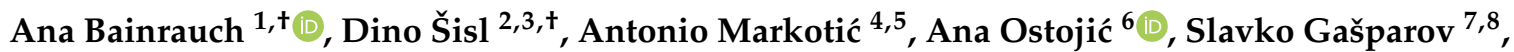 \\ Valerija Bralić Lang ${ }^{9}$, Nataša Kovačić ${ }^{2,10}$, Danka Grčević ${ }^{2,3}$, Anna Mrzljak ${ }^{6,7, *}$ and Tomislav Kelava ${ }^{2,3, *}$ \\ 1 Department of Internal Medicine, Merkur University Hospital, 10000 Zagreb, Croatia; \\ ana.bainrauch@gmail.com \\ 2 Laboratory for Molecular Immunology, Croatian Institute for Brain Research, University of Zagreb, \\ 10000 Zagreb, Croatia; dino.sisl@mef.hr (D.Š.); natasa.kovacic@mef.hr (N.K.); danka.grcevic@mef.hr (D.G.) \\ 3 Department of Physiology and Immunology, School of Medicine, University of Zagreb, 10000 Zagreb, Croatia \\ 4 Department of Physiology, School of Medicine, University of Mostar, 88000 Mostar, Bosnia and Herzegovina; \\ antoniomarkotic@msn.com \\ 5 Center for Clinical Pharmacology, University Clinical Hospital Mostar, \\ 88000 Mostar, Bosnia and Herzegovina \\ 6 Department of Gastroenterology and Hepatology, University Hospital Center Zagreb, University of Zagreb, \\ 10000 Zagreb, Croatia; ostojicana.zg@gmail.com \\ check for \\ updates \\ Citation: Bainrauch, A.; Šisl, D.; \\ Markotić, A.; Ostojić, A.; Gašparov, S.; \\ Bralić Lang, V.; Kovačić, N.; Grčević, \\ D.; Mrzljak, A.; Kelava, T. NOTCH3 \\ rs1043996 Polymorphism Is \\ School of Medicine, University of Zagreb, 10000 Zagreb, Croatia; gasprovslavko@gmail.com \\ 8 Department of Pathology and Cytology, Merkur University Hospital, 10000 Zagreb, Croatia \\ 9 Private Family Physician Office Zagreb, 10000 Zagreb, Croatia; valerija.bralic.lang@gmail.com \\ 10 Department of Anatomy, School of Medicine, University of Zagreb, 10000 Zagreb, Croatia \\ * Correspondence: anna.mrzljak@mef.hr (A.M.); tomislav.kelava@mef.hr (T.K.); \\ Tel.: +385-1-238-8888 (A.M.); +385-1-456-6945 (T.K.) \\ + Equal contribution.
} Associated with the Occurrence of Alcoholic Liver Cirrhosis Independently of PNPLA3 and TM6SF2 Polymorphisms. J. Clin. Med. 2021, 10, 4621. https://doi.org/ $10.3390 /$ jcm 10194621

Academic Editors: Hiroki Nishikawa and Mark W. Russo

Received: 16 August 2021

Accepted: 5 October 2021

Published: 8 October 2021

Publisher's Note: MDPI stays neutral with regard to jurisdictional claims in published maps and institutional affiliations.

Copyright: (c) 2021 by the authors. Licensee MDPI, Basel, Switzerland. This article is an open access article distributed under the terms and conditions of the Creative Commons Attribution (CC BY) license (https:/ / creativecommons.org/licenses/by/ $4.0 /)$.

\begin{abstract}
Alcoholic liver cirrhosis (ALC) is the most common indication for liver transplantation (LT) in Croatia and presents a risk factor for the development of hepatocellular carcinoma (HCC). However, genetic susceptibility has not yet been systematically studied. We aimed to investigate the contribution of the risk polymorphisms PNPLA3 rs738409, EGF rs4444903, TM6SF2 rs58542926, MTHFR rs1801133, previously identified in other populations and, additionally, the contribution of Notch-related polymorphisms (NOTCH1 rs3124591, NOTCH3 rs1043996 and rs1044116, NOTCH4 rs422951). The study included 401 patients. The ALC group consisted of 260 LT candidates, 128 of whom had histopathologically confirmed HCC, and 132 of whom were without HCC. The control group included 141 patients without liver disease. Genotyping was performed by PCR using Taqman assays. The patients' susceptibility to ALC was significantly associated with PNPLA3 rs738409, TM6SF2 rs58542926, and NOTCH3 rs1043996 polymorphisms. These polymorphisms remained significantly associated with ALC occurrence in a logistic regression model, even after additional model adjustment for sex and age. Cirrhotic patients with the PNPLA3 GG genotype demonstrated higher activity of ALT aminotransferases than patients with CC or CG genotypes. The susceptibility to the development of HCC in ALC was significantly associated with PNPLA3 rs738409 and EGF rs4444903 polymorphisms, and logistic regression confirmed these polymorphisms as independent predictors.
\end{abstract}

Keywords: single nucleotide polymorphisms; alcoholic liver disease; liver transplantation; hepatocellular carcinoma; Notch; PNPLA3

\section{Introduction}

End-stage alcoholic liver disease is one of the leading indications for liver transplantation (LT), and patients with alcoholic liver cirrhosis (ALC) are at higher risk for developing 
hepatocellular carcinoma (HCC) [1-3]. Genetic predisposition contributes to both liver fibrosis and its progression to HCC. The findings of both genome-wide association studies and candidate gene approach studies, conducted in the last decade, point to the patatin-like phospholipase domain-containing protein 3 (PNPLA3) rs738409 $\mathrm{G}$ allele and the transmembrane 6 superfamily member 2 (TM6SF2) rs58542926 T allele as the major genetic factors that increase susceptibility to the fibrosis [4-8]. These two single-nucleotide polymorphisms (SNPs) are also associated with the occurrence of HCC, while methylenetetrahydrofolate reductase (MTHFR) rs1801133 and epidermal growth factor (EGF) rs4444903 may further add to the HCC risk [9-13]. In Southeastern Europe, ALC is the most common indication for LT, but genetic susceptibility to it has not been systematically studied.

Recent studies on profibrotic and carcinogenic genes suggest that the evolutionarily conserved Notch signaling pathway may play a critical role in hepatic fibrogenesis and carcinogenesis [14-17]. Clinical studies have shown that Notch signaling is activated in liver samples from cirrhotic or HCC patients $[15,18]$. In addition, preclinical studies in various murine models showed that the inhibition of Notch signaling prevents fibrogenesis, while its overexpression promotes liver tumor formation in mice [18-20]. Therefore, Notchrelated molecules might be a promising target for the development of new antifibrotic and anticancer treatments [21,22]. Notch-related SNPs have been associated with an increased susceptibility to the development of breast cancer and poorer lung and liver cancer prognosis [23-25]. Still, their role as a risk factor for the development of cirrhosis or HCC has not yet been investigated.

In this study, we aimed to investigate the contribution of previously implicated genetic risk factors (PNPLA3, TM6SF2, EGF, MTHFR) for the development of cirrhosis and HCC in the Croatian population. Moreover, we introduced Notch-related SNPs to assess their potential role as risk factors. The association of the analyzed SNPs with clinical indices of disease severity was also determined as a secondary objective.

\section{Materials and Methods}

\subsection{Study Population and Data Collection}

After obtaining approval from the Merkur University Hospital and School of Medicine University of Zagreb Ethics Committee, the study included 401 patients. The ALC group consisted of 260 patients, 128 of whom had HCC, and 132 of whom were without HCC. The control group included 141 patients without liver disease. All the subjects were of Caucasian ethnicity and had no concomitant liver etiologies (viral, autoimmune or metabolic). In the ALC group, 253 patients received LT, and the presence or absence of HCC was confirmed by pathological examination of the explanted liver. In seven patients who did not undergo an LT, HCC was confirmed by radiological findings and examination of tissue obtained by liver biopsy. The data on alanine aminotransferase (ALT), aspartate aminotransferase (AST) activities, creatinine, alpha-fetoprotein (AFP) levels, tumor size, number of nodules, and microangioinvasion were obtained from the patients' hospital clinical records.

\subsection{DNA Isolation and Genotyping}

After obtaining written informed consent to participate in the study, venous blood samples were collected and stored at $-20{ }^{\circ} \mathrm{C}$ until DNA isolation. DNA was extracted from the whole blood samples $(200 \mathrm{uL})$ using the QIAGEN QIAamp DNA Blood Mini Kit spin method, according to the manufacturer's instructions. The DNA concentration and quality were determined using the NanoDrop ND1000 spectrophotometer, as described in previous studies [26]. All the samples were stored at $-20{ }^{\circ} \mathrm{C}$ until the batch genotype analysis. The genotypes were determined by polymerase chain reaction (PCR) using commercially available TaqMan SNP assays for PNPLA3 (rs738409), EGF (rs4444903), TM6SF2 (rs58542926), MTHFR (rs1801133), NOTCH1 (rs3124591), NOTCH3 (rs1043996 and rs1044116), and NOTCH4 (rs422951), using the ABI 7500 instrument (Applied Biosystems). All the SNPs were selected based on data from a haplotype map of the human genome 
(HapMap) with minor allele frequency (MAF) in the European population higher than $20 \%$, except for TM6SF2, for which the MAF was less than $20 \%$. However, the reason for its inclusion was its previously reported strong association with susceptibility to liver disease. The potential limitations of the results of the TM6SF2 analysis are addressed in detail in the Discussion section. The assay IDs and expected MAFs are provided in the Supporting information (Table A1 in Appendix A).

\subsection{Statistical Analysis}

Continuous variables are presented as median with interquartile range (IQR) or mean \pm standard deviation (SD) and compared using Mann-Whitney/Kruskal-Wallis test or Student's $t$-test/ANOVA, as appropriate. Categorical data were compared using the chi-square test. The genotype frequencies of all the polymorphisms were tested for Hardy-Weinberg equilibrium. For the genotype analysis we used dominant, recessive, codominant and log additive models. For the multiple comparison correction, the minimal false discovery rate level (Storey's q) was calculated [27]; results with both $p<0.05$ and $q<0.05$ were considered statistically significant. Multiple logistic regression was used to evaluate independent predictors of ALC or HCC development. The statistical analyses were performed using the free online software SNPStats (http:/ / bioinfo.iconcologia.net/snpstats accessed on: 14 August 2021) and R (a language and environment for statistical computing, $\mathrm{R}$ Foundation for Statistical Computing, Vienna, Austria), with the figures plotted in GraphPad Prism version 6 for Windows (GraphPad Software Inc., La Jolla, CA, USA).

\section{Results}

\subsection{Demographic, Laboratory and Histopathology Information}

Basic demographic, laboratory, and histopathology information on the patient groups are shown in Table 1 . The patients were predominantly males (89\%), without any statistically significant difference in age or sex between the groups. The patients with HCC had higher AFP and ALT levels and lower creatinine levels than the patients without HCC. The mean size of the HCC was $34.15 \pm 19.84 \mathrm{~mm}$. Microangioinvasion was found in $37.2 \%$ of the histopathological findings.

Table 1. Demographic, laboratory and histopathology information on different study groups.

\begin{tabular}{|c|c|c|c|c|}
\hline Characteristics & Controls & ALC & $\mathrm{ALC}+\mathrm{HCC}$ & $p$ Value \\
\hline $\mathrm{N}$ of patients & 141 & 132 & 128 & \\
\hline Age $^{1}$, years & $61.42 \pm 14.58$ & $60.24 \pm 6.27$ & $61.38 \pm 7.47$ & 0.57 \\
\hline \multicolumn{5}{|l|}{$\operatorname{Sex}^{2}$} \\
\hline $\mathrm{m}$ & $125(88.7 \%)$ & $116(87.9 \%)$ & $117(91.4 \%)$ & \multirow{2}{*}{0.63} \\
\hline $\mathrm{f}$ & $16(11.3 \%)$ & $16(12.1 \%)$ & $11(8.6 \%)$ & \\
\hline $\operatorname{ALT}(\mathrm{IU} / \mathrm{L})^{3}$ & & $23(18.5-33)$ & $32(24-45)$ & $2.4 \times 10^{-4}$ \\
\hline AST $(\mathrm{IU} / \mathrm{L})^{3}$ & & $47(36-63)$ & $50(39-70)$ & 0.92 \\
\hline $\begin{array}{l}\text { Creatinine } \\
(\mu \mathrm{mol} / \mathrm{L})^{3}\end{array}$ & & $91(66-127)$ & $79(63-101)$ & 0.011 \\
\hline $\begin{array}{l}\text { Platelets } \\
\left(\times 10^{9} / \mathrm{L}\right)^{3}\end{array}$ & & 87 (66.5-136.5) & $93(69-125)$ & 0.84 \\
\hline $\operatorname{AFP}(\mathrm{nmol} / \mathrm{L})^{3}$ & - & $3.8(2.9-5.35)$ & $7.3(4.3-20.7)$ & $3.5 \times 10^{-8}$ \\
\hline Tumor size (mm) & & & $34.15 \pm 19.84$ & - \\
\hline \multicolumn{5}{|l|}{ Angioinvasion 4} \\
\hline yes & & & $45(37.2 \%)$ & \\
\hline no & & & $76(62.8 \%)$ & - \\
\hline
\end{tabular}


Table 1. Cont.

\begin{tabular}{|c|c|c|c|c|}
\hline Characteristics & Controls & ALC & $\mathrm{ALC}+\mathrm{HCC}$ & $p$ Value \\
\hline \multicolumn{5}{|l|}{ Milan criteria 4} \\
\hline within & & & $63(52.1 \%)$ & \\
\hline beyond & & & $58(47.9 \%)$ & - \\
\hline
\end{tabular}

${ }_{1}^{1}$ mean \pm standard deviation; $p$ value was calculated by ANOVA and Bonferroni's post-hoc comparisons test ${ }^{2} p$ value was calculated by chi squared test. ${ }^{3}$ Median with interquartile range; $p$ value was calculated by Mann-Whitney-U test. ${ }^{4}$ Only for patients who received transplantation. AFP—alpha-fetoprotein; ALC—alcoholic liver cirrhosis; ALT—alanine aminotransferase; AST—aspartate transaminase.

\subsection{Assessment of Genetic Susceptibility to Alcoholic Cirrhosis}

All the genotypes were in Hardy-Weinberg equilibrium ( $p>0.05$ for all SNPs examined). We observed a linkage disequilibrium between the NOTCH3 rs1043996 and $\mathrm{rs} 1044116(\mathrm{r}=0.84)$. Weak linkage disequilibrium was present between the NOTCH3 and NOTCH1, as well as between NOTCH4 and EGF (Figure A1 in Appendix B).

PNPLA3 rs738409, TM6SF2 rs58542926 and NOTCH3 rs1043996 were significantly associated with susceptibility to cirrhosis, whereas no association was found for the remaining five SNPs examined. In a recessive model, the presence of the PNPLA3 GG genotype was markedly associated with a greater likelihood for liver cirrhosis development (OR $\left.95 \% \mathrm{CI}=4.03(1.93-8.41), p=8.4 \times 10^{-5}\right)$, and significant associations were also found in dominant, codominant and log additive models (Table 2). The presence of the $\mathrm{T}$ allele for TM6SF2 polymorphism was associated with greater odds of cirrhosis with OR 95\% CI $=2.86$ $(1.56-5.23)$ in the dominant model $\left(p=4.4 \times 10^{-4}\right)$. Finally, the NOTCH3 rs1043996 GG genotype was associated with a lower susceptibility to liver cirrhosis in a recessive model (OR $95 \%$ CI $=0.39(0.19-0.80, p=0.01)$. Detailed results for all the analyzed models are shown in Table 2.

Table 2. Single nucleotide polymorphism-associated odds ratio for cirrhosis occurrence.

\begin{tabular}{|c|c|c|c|c|c|c|}
\hline \multirow{2}{*}{ Gene SNP } & \multirow{2}{*}{ Model } & \multirow{2}{*}{ Genotype } & \multicolumn{2}{|c|}{ ALC } & \multirow{2}{*}{ OR } & \multirow{2}{*}{$p$} \\
\hline & & & No & Yes & & \\
\hline \multirow[t]{8}{*}{ PNPLA3 rs738409 } & Codominant & $\mathrm{CC}$ & $80(56.7 \%)$ & $82(31.5 \%)$ & 1.00 & \\
\hline & & CG & $52(36.9 \%)$ & $122(46.9 \%)$ & $2.29(1.46-3.58)$ & $4 \times 10^{-7}$ \\
\hline & & GG & $9(6.4 \%)$ & $56(21.5 \%)$ & $\begin{array}{c}6.07 \\
(2.82-13.09)\end{array}$ & \\
\hline & Dominant & $\mathrm{CC}$ & $80(56.7 \%)$ & $82(31.5 \%)$ & 1.00 & \\
\hline & & $\mathrm{CG}+\mathrm{GG}$ & $61(43.3 \%)$ & $178(68.5 \%)$ & $2.85(1.86-4.35)$ & $5.8 \times 10^{-6}$ \\
\hline & Recessive & $\mathrm{CC}+\mathrm{CG}$ & $132(93.6 \%)$ & $204(78.5 \%)$ & 1.00 & \\
\hline & & GG & $9(6.4 \%)$ & $56(21.5 \%)$ & $4.03(1.93-8.41)$ & $8.4 \times 10^{-5}$ \\
\hline & Log additive & & & & $2.39(1.73-3.32)$ & $6.3 \times 10^{-8}$ \\
\hline \multirow[t]{8}{*}{ EGF rs4444903 } & Codominant & AA & $49(34.8 \%)$ & $82(31.5 \%)$ & 1.00 & \\
\hline & & AG & $66(46.8 \%)$ & $133(51.1 \%)$ & $1.20(0.76-1.91)$ & 0.7 \\
\hline & & GG & $26(18.4 \%)$ & $45(17.3 \%)$ & $1.03(0.57-1.88)$ & \\
\hline & Dominant & AA & $49(34.8 \%)$ & $82(31.5 \%)$ & 1.00 & \\
\hline & & $\mathrm{AG}+\mathrm{GG}$ & $92(65.2 \%)$ & $178(68.5 \%)$ & $1.16(0.75-1.78)$ & 0.51 \\
\hline & Recessive & $\mathrm{AA}+\mathrm{AG}$ & $115(81.6 \%)$ & $215(82.7 \%)$ & 1.00 & \\
\hline & & GG & $26(18.4 \%)$ & $45(17.3 \%)$ & $0.93(0.54-1.58)$ & 0.78 \\
\hline & Log additive & & & & $1.04(0.78-1.40)$ & 0.77 \\
\hline \multirow[t]{8}{*}{ TM6SF2 rs58542926 } & Codominant & $\mathrm{CC}$ & $126(89.4 \%)$ & $194(74.6 \%)$ & 1.00 & \\
\hline & & $\mathrm{CT}$ & $15(10.6 \%)$ & $61(23.5 \%)$ & $2.64(1.44-4.85)$ & $7.6 \times 10^{-4}$ \\
\hline & & $\mathrm{TT}$ & $0(0 \%)$ & $5(1.9 \%)$ & NA & NA \\
\hline & Dominant & $\mathrm{CC}$ & $126(89.4 \%)$ & $194(74.6 \%)$ & 1.00 & \\
\hline & & $\mathrm{CT}+\mathrm{TT}$ & $15(10.6 \%)$ & $66(25.4 \%)$ & $2.86(1.56-5.23)$ & $4.4 \times 10^{-4}$ \\
\hline & Recessive & $\mathrm{CC}+\mathrm{CT}$ & $141(100 \%)$ & $255(98.1 \%)$ & 1.00 & \\
\hline & & $\mathrm{TT}$ & $0(0 \%)$ & $5(1.9 \%)$ & NA & NA \\
\hline & Log additive & & & & $2.83(1.58-5.07)$ & $3 \times 10^{-4}$ \\
\hline
\end{tabular}


Table 2. Cont.

\begin{tabular}{|c|c|c|c|c|c|c|}
\hline \multirow{2}{*}{ Gene SNP } & \multirow{2}{*}{ Model } & \multirow{2}{*}{ Genotype } & \multicolumn{2}{|c|}{ ALC } & \multirow{2}{*}{ OR } & \multirow{2}{*}{$p$} \\
\hline & & & No & Yes & & \\
\hline \multirow{8}{*}{ MTHFR rs1801133 } & \multirow{3}{*}{ Codominant } & GG & $58(41.1 \%)$ & $120(46.1 \%)$ & 1.00 & \multirow{3}{*}{0.46} \\
\hline & & AG & $64(45.4 \%)$ & $114(43.9 \%)$ & $0.86(0.56-1.33)$ & \\
\hline & & AA & $19(13.5 \%)$ & $26(10 \%)$ & $0.66(0.34-1.29)$ & \\
\hline & \multirow[t]{2}{*}{ Dominant } & GG & $58(41.1 \%)$ & $120(46.1 \%)$ & 1.00 & \multirow{3}{*}{0.33} \\
\hline & & $\mathrm{AG}+\mathrm{AA}$ & $83(58.9 \%)$ & $140(53.9 \%)$ & $0.82(0.54-1.23)$ & \\
\hline & \multirow[t]{2}{*}{ Recessive } & $\mathrm{GG}+\mathrm{AG}$ & $122(86.5 \%)$ & $234(90 \%)$ & 1.00 & \\
\hline & & AA & $19(13.5 \%)$ & $26(10 \%)$ & $0.71(0.38-1.34)$ & \multirow{2}{*}{$\begin{array}{c}0.3 \\
0.23\end{array}$} \\
\hline & Log additive & & & & $0.83(0.61-1.12)$ & \\
\hline \multirow[t]{8}{*}{ NOTCH1 rs3124591 } & \multirow[t]{3}{*}{ Codominant } & TT & $47(33.3 \%)$ & $79(30.4 \%)$ & 1.00 & \multirow{3}{*}{0.39} \\
\hline & & $\mathrm{CT}$ & $59(41.8 \%)$ & $127(48.9 \%)$ & $1.28(0.80-2.06)$ & \\
\hline & & $\mathrm{CC}$ & $35(24.8 \%)$ & $54(20.8 \%)$ & $0.92(0.53-1.60)$ & \\
\hline & \multirow[t]{2}{*}{ Dominant } & $\mathrm{TT}$ & $47(33.3 \%)$ & $79(30.4 \%)$ & 1.00 & \multirow{3}{*}{0.54} \\
\hline & & $\mathrm{CT}+\mathrm{CC}$ & $94(66.7 \%)$ & $181(69.6 \%)$ & $1.15(0.74-1.78)$ & \\
\hline & \multirow{2}{*}{ Recessive } & $\mathrm{TT}+\mathrm{CT}$ & $106(75.2 \%)$ & $206(79.2 \%)$ & 1.00 & \\
\hline & & $\mathrm{CC}$ & $35(24.8 \%)$ & $54(20.8 \%)$ & $0.79(0.49-1.29)$ & 0.35 \\
\hline & Log additive & & & & $0.98(0.74-1.30)$ & 0.88 \\
\hline \multirow[t]{8}{*}{ NOTCH3 rs1043996 } & \multirow[t]{3}{*}{ Codominant } & AA & $71(50.4 \%)$ & $144(55.4 \%)$ & 1.00 & \multirow{3}{*}{$0.036^{q}>0.05$} \\
\hline & & AG & $51(36.2 \%)$ & $101(38.9 \%)$ & $0.98(0.63-1.52)$ & \\
\hline & & GG & $19(13.5 \%)$ & $15(5.8 \%)$ & $0.39(0.19-0.81)$ & \\
\hline & \multirow[t]{2}{*}{ Dominant } & AA & $71(50.4 \%)$ & $144(55.4 \%)$ & 1.00 & \multirow{3}{*}{0.34} \\
\hline & & $\mathrm{AG}+\mathrm{GG}$ & $70(49.6 \%)$ & $116(44.6 \%)$ & $0.82(0.54-1.23)$ & \\
\hline & \multirow[t]{2}{*}{ Recessive } & $\mathrm{AA}+\mathrm{AG}$ & $122(86.5 \%)$ & $245(94.2 \%)$ & 1.00 & \\
\hline & & GG & $19(13.5 \%)$ & $15(5.8 \%)$ & $0.39(0.19-0.80)$ & 0.01 \\
\hline & Log additive & & & & $0.74(0.54-1.01)$ & 0.061 \\
\hline NOTCH3 rs1044116 & Codominant & $\mathrm{TT}$ & $84(59.6 \%)$ & $165(63.5 \%)$ & 1.00 & \\
\hline & & $\mathrm{CT}$ & $45(31.9 \%)$ & $84(32.3 \%)$ & $0.95(0.61-1.49)$ & 0.22 \\
\hline & & $\mathrm{CC}$ & $12(8.5 \%)$ & $11(4.2 \%)$ & $0.47(0.20-1.10)$ & \\
\hline & Dominant & $\mathrm{TT}$ & $84(59.6 \%)$ & $165(63.5 \%)$ & 1.00 & \\
\hline & & $\mathrm{CT}+\mathrm{CC}$ & $57(40.4 \%)$ & $95(36.5 \%)$ & $0.85(0.56-1.29)$ & 0.44 \\
\hline & Recessive & $\mathrm{TT}+\mathrm{CT}$ & $129(91.5 \%)$ & $249(95.8 \%)$ & 1.00 & \\
\hline & & $\mathrm{CC}$ & $12(8.5 \%)$ & $11(4.2 \%)$ & $0.47(0.20-1.11)$ & 0.086 \\
\hline & Log additive & & & & $0.80(0.57-1.12)$ & 0.20 \\
\hline NOTCH4 rs422951 & Codominant & $\mathrm{CC}$ & $33(23.4 \%)$ & $71(27.3 \%)$ & 1.00 & \\
\hline & & $\mathrm{CT}$ & $75(53.2 \%)$ & $128(49.2 \%)$ & $0.79(0.48-1.31)$ & 0.66 \\
\hline & & $\mathrm{TT}$ & $33(23.4 \%)$ & $61(23.5 \%)$ & $0.86(0.48-1.55)$ & \\
\hline & Dominant & $\mathrm{CC}$ & $33(23.4 \%)$ & $71(27.3 \%)$ & 1.00 & \\
\hline & & $\mathrm{CT}+\mathrm{TT}$ & $108(76.6 \%)$ & $189(72.7 \%)$ & $0.81(0.51-1.31)$ & 0.39 \\
\hline & Recessive & $\mathrm{CC}+\mathrm{CT}$ & $108(76.6 \%)$ & 199 (76.5\%) & 1.00 & \\
\hline & & $\mathrm{TT}$ & $33(23.4 \%)$ & $61(23.5 \%)$ & $1.00(0.62-1.63)$ & 0.99 \\
\hline & Log additive & & & & $0.92(0.69-1.24)$ & 0.6 \\
\hline
\end{tabular}

$q>0.05$ The difference did not reach statistically significant levels after correction for multiple comparison. ALC-alcoholic liver cirrhosis; $E G F$ - epidermal growth factor; MTHFR—-methylenetetrahydrofolate reductase; NA—not analyzed; not meaningful due to low minor allele frequency (see limitations in the Discussion section); OR_odds ratio; PNPLA3—patatin-like phospholipase domain-containing protein 3; TM6SF2-transmembrane 6 superfamily member 2.

The independence of predictors was further analyzed by multiple logistic regression. All three SNPs remained significantly associated with the susceptibility to cirrhosis, even after additional model adjustment for sex and age (Table 3). 
Table 3. Logistic regression analysis of predictors of cirrhosis development.

\begin{tabular}{|c|c|c|c|c|c|c|}
\hline \multirow{2}{*}{ Variables in the Model ${ }^{1}$} & \multirow{2}{*}{ b } & \multirow{2}{*}{ Wald } & \multirow{2}{*}{$p$} & \multirow{2}{*}{ OR $(95 \% C I)$} & \multicolumn{2}{|c|}{ Adjusted Model $^{2}$} \\
\hline & & & & & $p$ & OR $(95 \% C I)$ \\
\hline \multicolumn{7}{|l|}{ PNPLA3 (rs738409) } \\
\hline $\mathrm{C} / \mathrm{C}-\mathrm{C} / \mathrm{G}$ & reference & - & - & 1.00 & - & 1.00 \\
\hline $\mathrm{G} / \mathrm{G}$ & 1.47 & 14.2 & $1.6 \times 10^{-4}$ & $4.21(1.99-8.87)$ & $1.7 \times 10^{-4}$ & $4.20(1.99-8.87)$ \\
\hline \multicolumn{7}{|l|}{ TM6SF2 (rs58542926) } \\
\hline $\mathrm{C} / \mathrm{C}$ & reference & - & - & 1.00 & - & 1.00 \\
\hline $\mathrm{C} / \mathrm{T}-\mathrm{T} / \mathrm{T}$ & 1.06 & 11.5 & 0.001 & $2.89(1.56-5.35)$ & 0.001 & $2.89(1.57-5.35)$ \\
\hline \multicolumn{7}{|l|}{ NOTCH3 (rs1043996) } \\
\hline $\mathrm{A} / \mathrm{A}-\mathrm{A} / \mathrm{G}$ & reference & - & - & 1.00 & - & 1.00 \\
\hline GG & -0.82 & 4.75 & 0.029 & $0.44(0.21-0.92)$ & 0.025 & $0.43(0.20-0.90)$ \\
\hline
\end{tabular}

${ }^{1}$ PNPLA3, TM6SF2 and NOTCH3 polymorphisms were included in the model. ${ }^{2}$ Model additionally adjusted for sex and age. For each model $\mathrm{b}$ coefficient is shown with corresponding Wald statistic value, odds ratio with $95 \%$ confidence interval, and $p$ value. OR-odds ratio; PNPLA3 - patatin-like phospholipase domain-containing protein 3; TM6SF2 - transmembrane 6 superfamily member 2.

SNPs' Association with Pre-Transplantation Parameters

To further establish the role of the studied SNPs in the development of ALC, we analyzed their association with pre-transplantation parameters. Patients with the PNPLA3 GG genotype had significantly higher ALT activity in sera than patients with CC or CG genotype, while there was no difference in the creatinine concentration, platelet count or age of the patients at LT. None of the parameters differed between TM6SF2 genotypes. We found a higher platelet count in patients with the NOTCH3 rs1043996 GG ( $p=0.03)$ genotype, but the difference did not reach statistical significance after correcting for multiple comparison, and there was no significant difference in the other parameters that were examined (Figure 1). For the remaining five examined SNPs, the analysis did not reveal any statistically significant differences (data not shown).

\subsection{Assessment of Genetic Susceptibility to the Development of HCC in ALC Patients}

The analysis showed that patients with the PNPLA3 GG genotype are more susceptible to HCC occurrence. A significant association was found in the recessive model (OR $95 \% \mathrm{CI}=2.96(1.57-5.58), p<0.001)$ and in the codominant and log-additive models. The results did not show any significant associations for the other two SNPs that were significantly associated with the susceptibility to cirrhosis (TM6SF2 rs58542926 and NOTCH3 rs1043996). On the other hand, the SNP for EGF, which was not associated with a risk of cirrhosis, was significantly associated with an increased likelihood of HCC progression in the dominant model (OR 95\%CI = 1.97 (1.15-3.36), $p=0.012$, for GG/AG vs. AA genotype). No significant association with HCC was found for any other SNP (detailed results are presented in Table 4). Multiple logistic regression revealed that SNPs for the PNPLA3 and EGF genes are independent predictors of the HCC development (Table 5).

Association of SNPs with HCC-Related Parameters

There was no association between HCC-related parameters (tumor size, microangionvasion, number of nodules or AFP levels) and the SNPs of the genes significantly associated with HCC occurrence (PNPLA3 and EGF, Figure 2), or between any of the other polymorphisms studied (data not shown). 
A

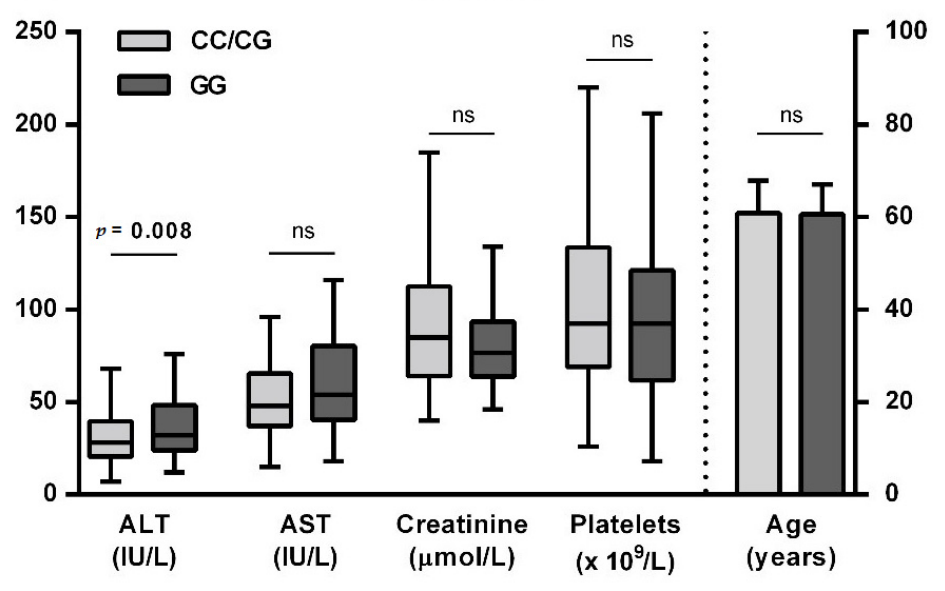

B

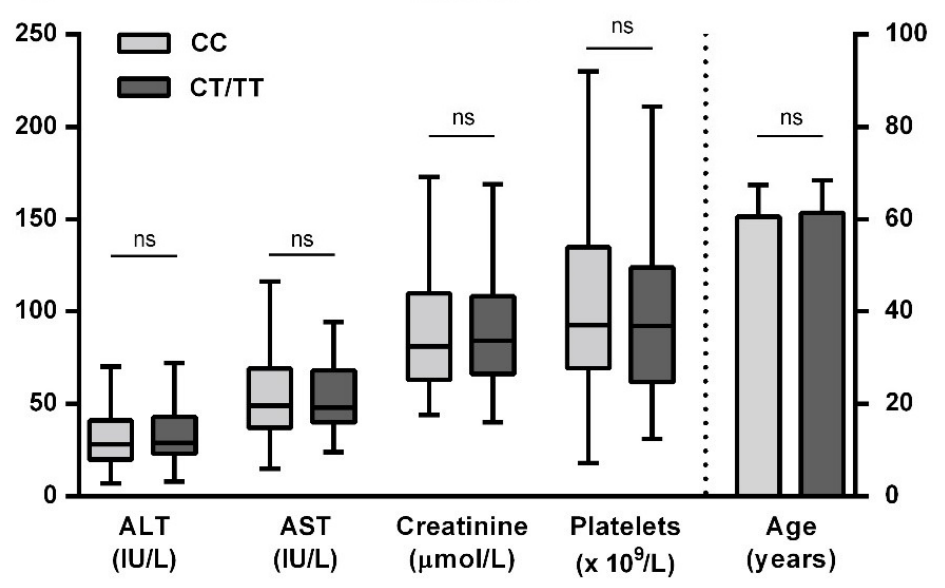

C

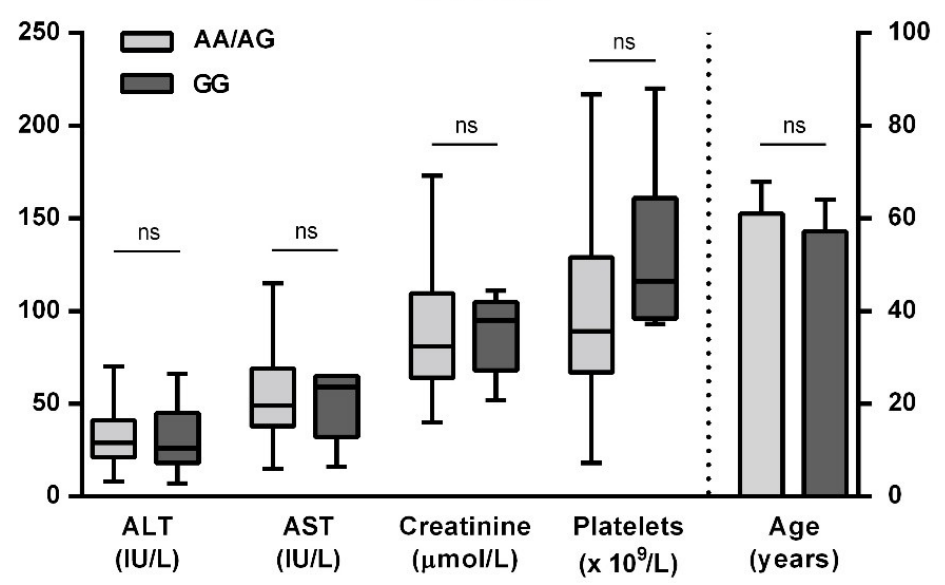

Figure 1. Association of SNPs with pre-transplantation parameters. Comparisons for (A) PNPLA3, (B) TM6SF2, and (C) NOTCH3 rs1043996 genotypes are shown. Alanine aminotransferase (ALT), aspartate transaminase (AST), creatinine levels, and platelets were not normally distributed and are presented as median with interquartile range; the Mann-Whitney-U test was used for statistical comparison. Age at transplantation was normally distributed; therefore, data are presented as mean with standard deviation and the Student's $t$-test was used for statistical comparison. ALTalanine aminotransferase; AST—aspartate transaminase; ns—not significant; PNPLA3 — patatin-like phospholipase domain-containing protein 3; TM6SF2-transmembrane 6 superfamily member 2. 
Table 4. Single-nucleotide polymorphism-associated odds ratio for HCC occurrence.

\begin{tabular}{|c|c|c|c|c|c|c|}
\hline \multirow{2}{*}{ Gene SNP } & \multirow{2}{*}{ Model } & \multirow{2}{*}{ Genotype } & \multicolumn{2}{|c|}{ HCC } & \multirow{2}{*}{ OR } & \multirow{2}{*}{$p$} \\
\hline & & & No & Yes & & \\
\hline \multirow[t]{8}{*}{ PNPLA3 rs738409 } & Codominant & $\mathrm{CC}$ & $47(35.6 \%)$ & $35(27.3 \%)$ & 1.00 & \\
\hline & & CG & $68(51.5 \%)$ & $54(42.2 \%)$ & 1.07 (0.61-1.88) & 0.002 \\
\hline & & GG & $17(12.9 \%)$ & $39(30.5 \%)$ & $3.08(1.50-6.32)$ & \\
\hline & Dominant & $\mathrm{CC}$ & $47(35.6 \%)$ & $35(27.3 \%)$ & 1.00 & \\
\hline & & $\mathrm{CG}+\mathrm{GG}$ & $85(64.4 \%)$ & $93(72.7 \%)$ & $1.47(0.87-2.49)$ & 0.15 \\
\hline & Recessive & $\mathrm{CC}+\mathrm{CG}$ & 115 (87.1\%) & $89(69.5 \%)$ & 1.00 & \\
\hline & & GG & $17(12.9 \%)$ & $39(30.5 \%)$ & $2.96(1.57-5.58)$ & $5.6 \times 10^{-4}$ \\
\hline & Log additive & & & & $1.66(1.17-2.35)$ & 0.004 \\
\hline \multirow[t]{8}{*}{ EGF rs4444903 } & Codominant & AA & $51(38.6 \%)$ & $31(24.2 \%)$ & 1.00 & \\
\hline & & AG & $61(46.2 \%)$ & $72(56.3 \%)$ & $1.94(1.11-3.41)$ & $0.042 q>0.05$ \\
\hline & & GG & $20(15.2 \%)$ & $25(19.5 \%)$ & $2.06(0.98-4.30)$ & \\
\hline & Dominant & $\mathrm{AA}$ & $51(38.6 \%)$ & $31(24.2 \%)$ & 1.00 & \\
\hline & & $\mathrm{AG}+\mathrm{GG}$ & $81(61.4 \%)$ & $97(75.8 \%)$ & $1.97(1.15-3.36)$ & 0.012 \\
\hline & Recessive & $\mathrm{AA}+\mathrm{AG}$ & $112(84.8 \%)$ & $103(80.5 \%)$ & 1.00 & \\
\hline & & GG & $20(15.2 \%)$ & $25(19.5 \%)$ & $1.36(0.71-2.59)$ & 0.35 \\
\hline & Log additive & & & & $1.50(1.05-2.16)$ & $0.026^{q>0.05}$ \\
\hline \multirow[t]{8}{*}{ TM6SF2 rs58542926 } & Codominant & $\mathrm{CC}$ & $100(75.8 \%)$ & $94(73.4 \%)$ & 1.00 & \\
\hline & & $\mathrm{CT}$ & $31(23.5 \%)$ & $30(23.4 \%)$ & $1.03(0.58-1.83)$ & 0.36 \\
\hline & & $\mathrm{TT}$ & $1(0.8 \%)$ & $4(3.1 \%)$ & $\begin{array}{c}4.26 \\
(0.47-38.72)\end{array}$ & \\
\hline & Dominant & $\mathrm{CC}$ & $100(75.8 \%)$ & $94(73.4 \%)$ & 1.00 & \\
\hline & & $\mathrm{CT}+\mathrm{TT}$ & $32(24.2 \%)$ & $34(26.6 \%)$ & $1.13(0.65-1.98)$ & 0.67 \\
\hline & Recessive & $\mathrm{CC}+\mathrm{CT}$ & $131(99.2 \%)$ & $124(96.9 \%)$ & 1.00 & \\
\hline & & $\mathrm{TT}$ & $1(0.8 \%)$ & $4(3.1 \%)$ & $\begin{array}{c}4.23 \\
(0.47-38.29)\end{array}$ & 0.15 \\
\hline & Log additive & & & & $1.22(0.74-2.01)$ & 0.44 \\
\hline \multirow[t]{8}{*}{ MTHFR rs1801133 } & Codominant & GG & $57(43.2 \%)$ & $63(49.2 \%)$ & 1.00 & \\
\hline & & AG & $61(46.2 \%)$ & $53(41.4 \%)$ & $0.79(0.47-1.31)$ & 0.62 \\
\hline & & AA & $14(10.6 \%)$ & $12(9.4 \%)$ & $0.78(0.33-1.81)$ & \\
\hline & Dominant & GG & $57(43.2 \%)$ & $63(49.2 \%)$ & 1.00 & \\
\hline & & $\mathrm{AG}+\mathrm{AA}$ & $75(56.8 \%)$ & $65(50.8 \%)$ & $0.78(0.48-1.28)$ & 0.33 \\
\hline & Recessive & $\mathrm{GG}+\mathrm{AG}$ & $118(89.4 \%)$ & $116(90.6 \%)$ & 1.00 & \\
\hline & & $\mathrm{AA}$ & $14(10.6 \%)$ & $12(9.4 \%)$ & $0.87(0.39-1.96)$ & 0.74 \\
\hline & Log additive & & & & $0.84(0.58-1.22)$ & 0.37 \\
\hline \multirow[t]{8}{*}{ NOTCH1 rs3124591 } & Codominant & TT & $40(30.3 \%)$ & $39(30.5 \%)$ & 1.00 & \\
\hline & & $\mathrm{CT}$ & $63(47.7 \%)$ & $64(50.0 \%)$ & $1.04(0.59-1.83)$ & 0.88 \\
\hline & & $\mathrm{CC}$ & $29(22.0 \%)$ & $25(19.5 \%)$ & $0.88(0.44-1.77)$ & \\
\hline & Dominant & $\mathrm{TT}$ & $40(30.3 \%)$ & $39(30.5 \%)$ & 1.00 & \\
\hline & & $\mathrm{CT}+\mathrm{CC}$ & $92(69.7 \%)$ & $89(69.5 \%)$ & $0.99(0.58-1.68)$ & 0.98 \\
\hline & Recessive & $\mathrm{TT}+\mathrm{CT}$ & $103(78.0 \%)$ & $103(80.5 \%)$ & 1.00 & \\
\hline & & $\mathrm{CC}$ & $29(22.0 \%)$ & $25(19.5 \%)$ & $0.86(0.47-1.57)$ & 0.63 \\
\hline & Log additive & & & & $0.95(0.67-1.34)$ & 0.77 \\
\hline \multirow[t]{8}{*}{ NOTCH3 rs1043996 } & Codominant & AA & $74(56.1 \%)$ & $70(54.7 \%)$ & 1.0 & \\
\hline & & $\mathrm{AG}$ & $54(40.9 \%)$ & $47(36.7 \%)$ & $0.92(0.55-1.53)$ & 0.14 \\
\hline & & GG & $4(3 \%)$ & $11(8.6 \%)$ & $2.91(0.88-9.56)$ & \\
\hline & Dominant & AA & $74(56.1 \%)$ & $70(54.7 \%)$ & 1.00 & \\
\hline & & $A G+G G$ & $58(43.9 \%)$ & $58(45.3 \%)$ & $1.06(0.65-1.72)$ & 0.82 \\
\hline & Recessive & $\mathrm{AA}+\mathrm{AG}$ & $128(97 \%)$ & $117(91.4 \%)$ & 1.00 & \\
\hline & & GG & $4(3 \%)$ & $11(8.6 \%)$ & $3.01(0.93-9.71)$ & 0.06 \\
\hline & Log additive & & & & $1.21(0.81-1.81)$ & 0.35 \\
\hline
\end{tabular}


Table 4. Cont.

\begin{tabular}{|c|c|c|c|c|c|c|}
\hline \multirow{2}{*}{ Gene SNP } & \multirow{2}{*}{ Model } & \multirow{2}{*}{ Genotype } & \multicolumn{2}{|c|}{ HCC } & \multirow{2}{*}{ OR } & \multirow{2}{*}{$p$} \\
\hline & & & No & Yes & & \\
\hline \multirow[t]{8}{*}{ NOTCH3 rs1044116 } & \multirow{3}{*}{ Codominant } & $\mathrm{TT}$ & $84(63.6 \%)$ & $81(63.3 \%)$ & 1.00 & \multirow{3}{*}{0.25} \\
\hline & & $\mathrm{CT}$ & $45(34.1 \%)$ & $39(30.5 \%)$ & $0.90(0.53-1.52)$ & \\
\hline & & $\mathrm{CC}$ & $3(2.3 \%)$ & $8(6.2 \%)$ & $\begin{array}{c}2.77 \\
(0.71-10.79)\end{array}$ & \\
\hline & \multirow[t]{2}{*}{ Dominant } & $\mathrm{TT}$ & $84(63.6 \%)$ & $81(63.3 \%)$ & 1.00 & \multirow{3}{*}{0.95} \\
\hline & & $\mathrm{CT}+\mathrm{CC}$ & $48(36.4 \%)$ & $47(36.7 \%)$ & $1.02(0.61-1.68)$ & \\
\hline & \multirow[t]{2}{*}{ Recessive } & $\mathrm{TT}+\mathrm{CT}$ & $129(97.7 \%)$ & $120(93.8 \%)$ & 1.00 & \\
\hline & & $\mathrm{CC}$ & $3(2.3 \%)$ & $8(6.2 \%)$ & $\begin{array}{c}2.87 \\
(0.74-11.06)\end{array}$ & 0.11 \\
\hline & Log additive & & & & $1.14(0.75-1.75)$ & 0.54 \\
\hline \multirow[t]{8}{*}{ NOTCH4 rs422951 } & \multirow[t]{3}{*}{ Codominant } & $\mathrm{CC}$ & $40(30.3 \%)$ & $31(24.2 \%)$ & 1.0 & \multirow{3}{*}{0.53} \\
\hline & & $\mathrm{CT}$ & $63(47.7 \%)$ & $65(50.8 \%)$ & $1.33(0.74-2.39)$ & \\
\hline & & $\mathrm{TT}$ & $29(22.0 \%)$ & $32(25.0 \%)$ & $1.42(0.72-2.83)$ & \\
\hline & \multirow[t]{2}{*}{ Dominant } & $\mathrm{CC}$ & $40(30.3 \%)$ & $31(24.2 \%)$ & 1.00 & \multirow{3}{*}{0.27} \\
\hline & & $\mathrm{CT}+\mathrm{TT}$ & $92(69.7 \%)$ & $97(75.8 \%)$ & $1.36(0.79-2.36)$ & \\
\hline & \multirow[t]{2}{*}{ Recessive } & $\mathrm{CC}+\mathrm{CT}$ & $103(78.0 \%)$ & $96(75.0 \%)$ & 1.00 & \\
\hline & & TT & $29(22.0 \%)$ & $32(25.0 \%)$ & $1.18(0.67-2.10)$ & 0.56 \\
\hline & Log additive & & & & $1.20(0.85-1.69)$ & 0.3 \\
\hline
\end{tabular}

$q>0.05$ The difference did not reach statistically significant levels after correction for multiple comparison. EGF-epidermal growth factor; MTHFR - methylenetetrahydrofolate reductase; OR—odds ratio; PNPLA3—patatin-like phospholipase domain-containing protein 3; TM6SF2-transmembrane 6 superfamily member 2 .

\section{PNPLA3}
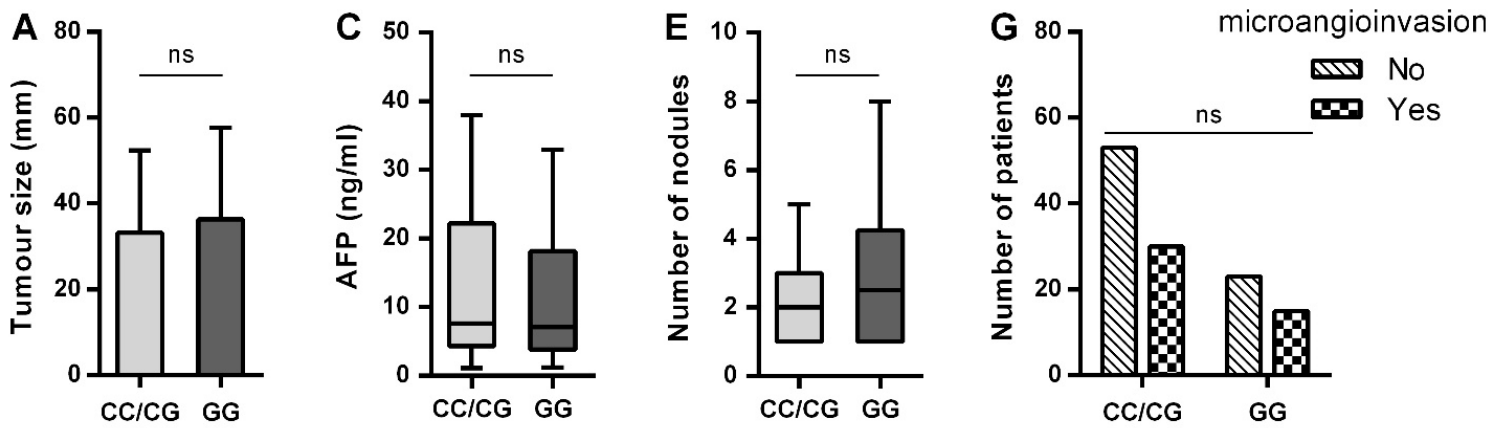

EGF
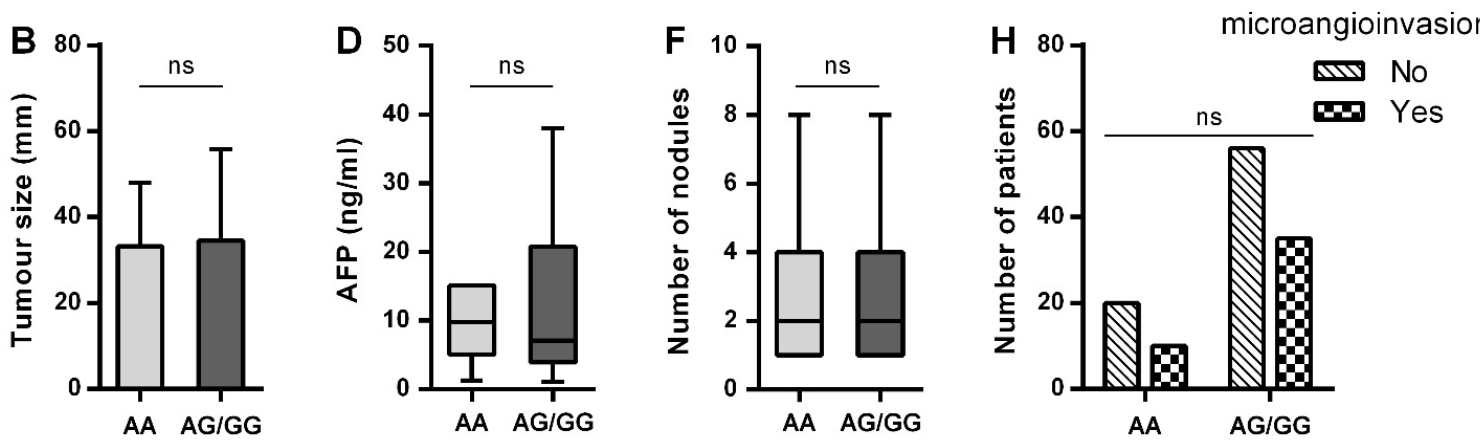

Figure 2. Association of SNPs with HCC-related parameters. Tumor size (A,B), alpha-fetoprotein levels (C,D), and number of nodules $(\mathbf{E}, \mathbf{F})$ are presented as median with interquartile range; the Mann-Whitney-U test was used for statistical comparison. For microangioinvasion $(\mathbf{G}, \mathbf{H})$, the number of patients is shown, and the chi-square test was used for statistical comparison. AFP—alpha-fetoprotein; EGF-epidermal growth factor; ns—not significant; PNPLA3-patatin-like phospholipase domain-containing protein 3. 
Table 5. Logistic regression analysis of predictors for hepatocellular carcinoma development.

\begin{tabular}{|c|c|c|c|c|c|c|}
\hline \multirow{2}{*}{ Variables in the Model ${ }^{1}$} & \multirow{2}{*}{$\mathbf{b}$} & \multirow{2}{*}{ Wald } & \multirow{2}{*}{$p$} & \multirow{2}{*}{ OR $(95 \% C I)$} & \multicolumn{2}{|c|}{ Adjusted Model $^{2}$} \\
\hline & & & & & $p$ & OR $(95 \% \mathrm{CI})$ \\
\hline \multicolumn{7}{|l|}{ PNPLA3 (rs738409) } \\
\hline $\mathrm{C} / \mathrm{C}-\mathrm{C} / \mathrm{G}$ & reference & - & - & 1.00 & - & 1.00 \\
\hline $\mathrm{G} / \mathrm{G}$ & 1.12 & 11.7 & 0.001 & $3.07(1.61-5.85)$ & 0.001 & $3.10(1.62-5.92)$ \\
\hline \multicolumn{7}{|l|}{ EGF (rs4444903) } \\
\hline $\mathrm{A} / \mathrm{A}$ & reference & - & - & 1.00 & - & 1.00 \\
\hline $\mathrm{A} / \mathrm{G}-\mathrm{G} / \mathrm{G}$ & 0.72 & 6.57 & 0.010 & $2.06(1.19-3.57)$ & 0.013 & $2.01(1.16-3.51)$ \\
\hline
\end{tabular}

\section{Discussion}

This is the first study to systematically examine the genetic susceptibility to ALC in the Southeast European population, where ALC is the leading cause of LT. A strength of this study is that it included age- and sex-matched patients who underwent LT and were diagnosed with HCC by histopathologic examination, which is superior to the radiologic diagnosis and/or mathematical correction for age usually performed in similar studies. Data from previous studies conducted in different populations showed a consistent association between PNPLA3 and TM6SF2 polymorphisms and susceptibility to ALC [4-8,28,29]. The study shows that they are also important risk factors in Croatia. To further establish the additional genetic risk factors for ALC, we investigated the possible contribution of polymorphisms in Notch receptors and found that NOTCH3 rs1043996 GG carriers have a reduced probability of developing ALC that is independent of the PNPLA3 or TM6SF2 genotypes. Yu et al. [25] reported a significant association between $\mathrm{NOTCH} 3$ and NOTCH4 SNPs with HCC survival, but to the best of our knowledge, the association with ALC or HCC incidence has not been studied. Notch signaling mediates communication between neighboring cells to guide cell fate decisions both during embryogenesis and postnatal life. In the liver, Notch signaling is required for normal development, but recent findings have also identified Notch activation as an essential contributor to fibrogenesis in several murine models of fibrosis, and the overexpression of Notch has been confirmed in human samples of cirrhotic liver tissue as well as in liver cancer $[15,16,18,20,30,31]$.

Patients with ALC have a higher risk of developing HCC, with a cumulative 5-year risk of approximately $8 \%$ [32]. The activation of the Notch signaling pathway has recently been linked to the occurrence of HCC [33]. We found an association between the PNPLA3 and EGF polymorphisms and susceptibility to HCC, but no association was found for MTHFR, TM6SF2, or Notch-related polymorphisms. Tanabe et al. [34] reported that EGF rs4444903 is associated with HCC and found higher EGF secretion in carriers of the G allele. Later studies, mainly in patients with hepatitis $C$ virus-related cirrhosis, confirmed this finding, but independence from PNPLA3, a dominant risk factor, was not tested [35,36]. Our results confirm EGF as a risk factor in ALC-related HCC and logistic regression shows its independence from PNPLA3. Furthermore, we show that $E G F$ is not a risk factor for the development of ALC, which has not been previously investigated, to the best of our knowledge. Compared to the CC genotype, heterozygosity for TM6SF2 (CT genotype) was clearly associated with a higher probability of developing ALC, but it was not associated with progression to HCC $(\mathrm{OR} 95 \% \mathrm{CI}=1.03(0.58-1.83), p=0.36)$. Due to the low incidence of the TT genotype in the TM6SF2 gene, the study was unable to assess its association with ALC or HCC; however, four patients with this genotype had HCC, one patient had ALC, and no patients in the control group had this genotype, which suggests a potential link. Therefore, the TT genotype for TM6SF2 may be a risk factor for a small number of patients, but its role needs to be further confirmed by a meta-analysis of a large number of studies.

Previous studies also suggested a possible role of MTHFR rs1801133 in HCC progression, but further research showed conflicting results. This includes two recent meta- 
analyses, one of which confirmed the association, while the other rejected it, with the exception of the Asian subpopulation [9,11,37]. Recently, Pineda-Tenor et al. [38] reported that MTHFR rs1801133 polymorphism is associated with the progression of liver fibrosis in chronic hepatitis C. However, we found no association between the MTHFR polymorphism and susceptibility to ALC or HCC.

In addition, we found higher activity of ALT aminotransferases in the sera of GG PNPLA3 carriers, but there was no significant association between the PNPLA3 genotype and the patient's age at transplantation or HCC-related prognostic factors, such as tumor size and microangioinvasion. This is in line with the results of the study by Khlaiphuengsin et al. [39], which did not find any relationship between the PNPLA3 genotype and tumor clinical characteristics. In addition, the results did not show a significant association between other examined SNPs and indices of disease severity.

\section{Conclusions}

This study showed that the NOTCH3 rs1043996 GG genotype is associated with lower susceptibility to ALC, independently of PNPLA3 and TM6SF2 polymorphisms. The occurrence of HCC in ALC was associated with PNPLA3 and EGF polymorphisms, and none of the Notch-related SNPs were significantly associated with susceptibility to HCC.

Author Contributions: Conceptualization, D.G., A.M. (Anna Mrzljak) and T.K.; data curation, A.B., D.Š., A.O. and V.B.L.; formal analysis, A.M. (Antonio Markotić), A.M. (Anna Mrzljak) and T.K.; funding acquisition, N.K., D.G. and T.K.; investigation, A.B., D.Š., A.M. (Antonio Markotić), A.O., S.G. and V.B.L.; methodology, A.B., D.Š., S.G., V.B.L., N.K. and T.K.; resources, N.K., D.G., A.M. (Anna Mrzljak) and T.K.; Software, A.M. (Antonio Markotić); validation, S.G., V.B.L., D.G. and T.K.; visualization, A.O.; writing—original draft, A.B. and D.Š.; writing—review and editing, A.M. (Antonio Markotić), S.G., N.K., D.G., A.M. (Anna Mrzljak) and T.K. All authors have read and agreed to the published version of the manuscript.

Funding: This research was funded by Croatian Science Foundation grants: UIP-2017-05-1965 and IP-2018-01-2414: “The Role of Notch Signaling Pathway in Pathogenesis of Hepatic Fibrosis" (grant number UIP-2017-05-1965 to T Kelava) and "Notch signaling in osteoclast progenitors induced by rheumatoid arthritis" (grant number IP-2018-01-2414 to D Grcevic).

Institutional Review Board Statement: The study was conducted according to the guidelines of the Declaration of Helsinki and reviewed and approved by the Merkur University Hospital (Case number: 0311-11070) and the School of Medicine University of Zagreb Ethics Committees (Case number 380-59-10106-18-111/251).

Informed Consent Statement: Informed consent was obtained from all subjects involved in the study.

Data Availability Statement: Data are contained within the article. Any additional data are available from the corresponding author upon reasonable request.

Conflicts of Interest: The authors declare no conflict of interest.

\section{Appendix A}

Table A1. Assay IDs and expected minor allele frequency for the selected single-nucleotide polymorphisms.

\begin{tabular}{|c|c|c|c|}
\hline SNP ID & Gene & Assay ID & Expected MAF ${ }^{1}$ \\
\hline rs738409 C>G & PNPLA3 & 7241_10 & $22.5 \%$ \\
\hline rs4444903 A>G & $E G F$ & C__27031637_30 & $39 \%$ \\
\hline rs58542926 C>T & TM6SF26 & C__89463510_10 & $7 \%$ \\
\hline rs1801133 G>A & MTHFR & C__1202883_20 & $37 \%$ \\
\hline rs3124591 T>C & NOTCH1 & C___189059_10 & $50 \%$ \\
\hline
\end{tabular}


Table A1. Cont.

\begin{tabular}{cccc}
\hline SNP ID & Gene & Assay ID & Expected MAF \\
\hline rs1043996 A $>$ G & NOTCH3 & C_22275447_10 & $31 \%$ \\
\hline rs1044116 T $>$ C & NOTCH3 & C__7494184_10 & 24\% \\
\hline rs422951 C $>$ T & NOTCH4 & C__3293790_10 & $46 \%$ \\
\hline
\end{tabular}

${ }^{1}$ from the data for the European population in the SNP database, available at: https://www.ncbi.nlm.nih gov/snp/ (accessed on 14 August 2021). EGF-epidermal growth factor; MAF-minor allele frequency; MTHFR - methylenetetrahydrofolate reductase; PNPLA3-patatin-like phospholipase domain-containing protein 3; TM6SF2-transmembrane 6 superfamily member 2.

\section{Appendix B}

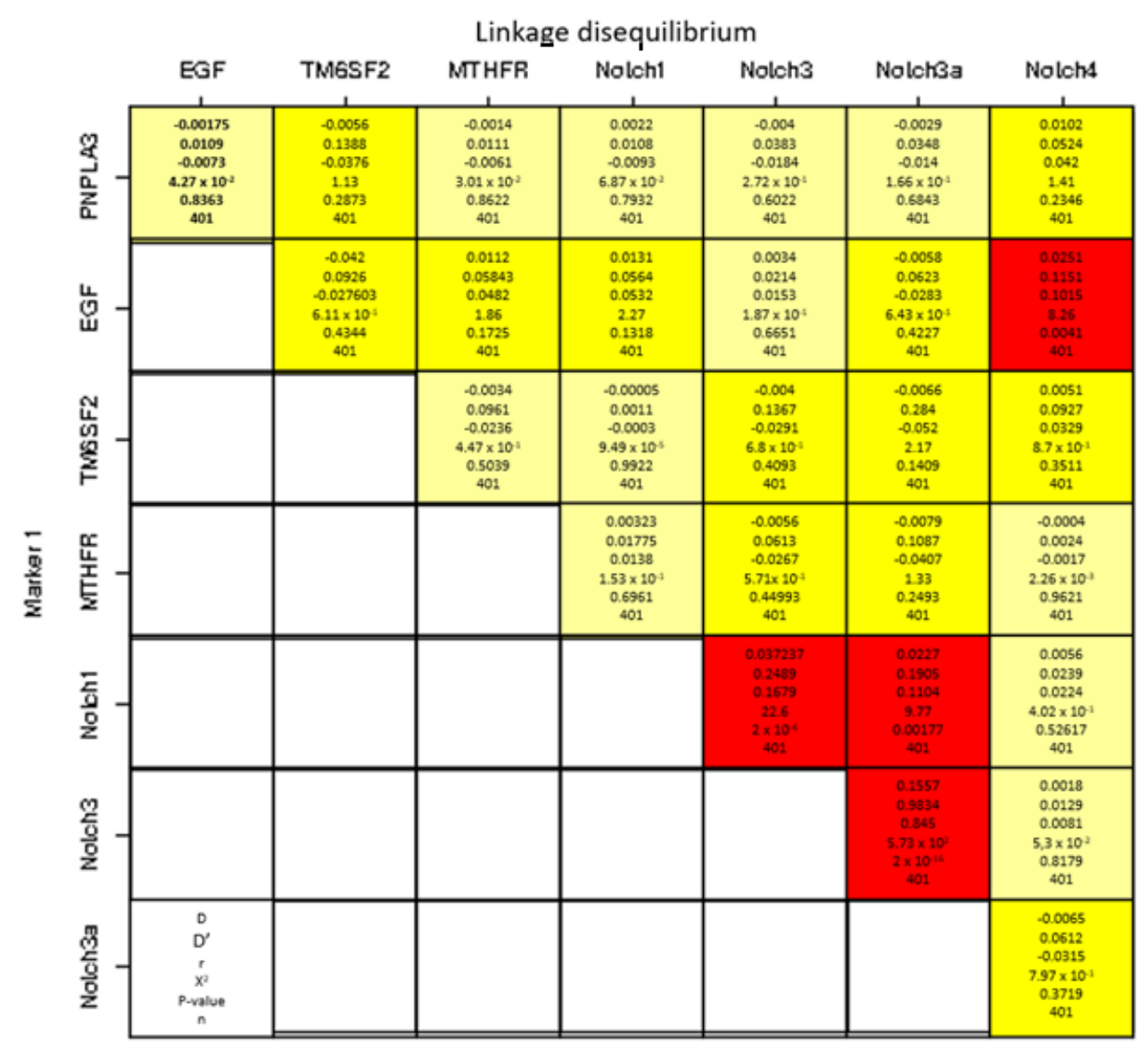

Marker 2

Figure A1. Linkage disequilibrium for the examined single-nucleotide polymorphisms. Analysis was performed using the free online software SNPStats (http://bioinfo.iconcologia.net/snpstats, accessed on: 14 August 2021). EGF rs4444903; MTHFR rs1801133; NOTCH1 rs3124591; NOTCH3 rs1043996; NOTCH3a rs1044116; NOTCH4 rs422951; PNPLA3 rs738409; TM6SF26 rs58542926.

\section{References}

1. Marroni, C.A.; Fleck, A.M., Jr.; Fernandes, S.A.; Galant, L.H.; Mucenic, M.; Meine, M.H.d.M.; Mariante-Neto, G.; Brandão, A.B.d.M. Liver transplantation and alcoholic liver disease: History, controversies, and considerations. World J. Gastroenterol. 2018, 24, 2785-2805. [CrossRef] [PubMed]

2. Singal, A.G.; Lampertico, P.; Nahon, P. Epidemiology and surveillance for hepatocellular carcinoma: New trends. J. Hepatol. 2020, 72, 250-261. [CrossRef]

3. Ganne-Carrié, N.; Nahon, P. Hepatocellular carcinoma in the setting of alcohol-related liver disease. J. Hepatol. 2019, 70, $284-293$. [CrossRef] [PubMed] 
4. Schwantes-An, T.; Darlay, R.; Mathurin, P.; Masson, S.; Liangpunsakul, S.; Mueller, S.; Aithal, G.P.; Eyer, F.; Gleeson, D.; Thompson, A.; et al. Genome-wide Association Study and Meta-analysis on Alcohol-Associated Liver Cirrhosis Identifies Genetic Risk Factors. Hepatology 2020, 73, 1920-1931. [CrossRef] [PubMed]

5. Buch, S.S.; Stickel, F.; Trépo, E.; Way, M.; Herrmann, A.; Nischalke, H.D.; Brosch, M.; Rosendahl, J.J.; Berg, T.; Ridinger, M.M.; et al. A genome-wide association study confirms PNPLA3 and identifies TM6SF2 and MBOAT7 as risk loci for alcohol-related cirrhosis. Nat. Genet. 2015, 47, 1443-1448. [CrossRef]

6. Stickel, F.; Buch, S.; Nischalke, H.D.; Weiss, K.H.; Gotthardt, D.; Fischer, J.; Rosendahl, J.; Marot, A.; Elamly, M.; Casper, M.; et al. Genetic variants in PNPLA3 and TM6SF2 predispose to the development of hepatocellular carcinoma in individuals with alcohol-related cirrhosis. Am. J. Gastroenterol. 2018, 113, 1475-1483. [CrossRef]

7. Yang, J.; Trépo, E.; Nahon, P.; Cao, Q.; Moreno, C.; Letouzé, E.; Imbeaud, S.; Gustot, T.; Deviere, J.; Debette, S.; et al. PNPLA3 and TM6SF2 variants as risk factors of hepatocellular carcinoma across various etiologies and severity of underlying liver diseases. Int. J. Cancer 2018, 144, 533-544. [CrossRef] [PubMed]

8. Falleti, E.; Cussigh, A.; Cmet, S.; Fabris, C.; Toniutto, P. PNPLA3 rs738409 and TM6SF2 rs58542926 variants increase the risk of hepatocellular carcinoma in alcoholic cirrhosis. Dig. Liver Dis. 2015, 48, 69-75. [CrossRef]

9. $\mathrm{Su}, \mathrm{H}$. Correlation Between MTHFR Polymorphisms and Hepatocellular Carcinoma: A Meta-analysis. Nutr. Cancer 2019, 71, 1055-1060. [CrossRef]

10. Wang, C.; Xie, H.; Lu, D.; Ling, Q.; Jin, P.; Li, H.; Zhuang, R.; Xu, X.; Zheng, S. The MTHFR polymorphism affect the susceptibility of HCC and the prognosis of HCC liver transplantation. Clin. Transl. Oncol. 2017, 20, 448-456. [CrossRef]

11. Qin, X.; Peng, Q.; Chen, Z.; Deng, Y.; Huang, S.; Xu, J.; Li, H.; Li, S.; Zhao, J. The Association between MTHFR Gene Polymorphisms and Hepatocellular Carcinoma Risk: A Meta-Analysis. PLoS ONE 2013, 8, e56070. [CrossRef]

12. Jiang, G.; Yu, K.; Shao, L.; Yu, X.; Hu, C.; Qian, P.; Xie, H.; Li, J.; Zheng, J.; Zheng, S. Association between epidermal growth factor gene $+61 \mathrm{~A} / \mathrm{G}$ polymorphism and the risk of hepatocellular carcinoma: A meta-analysis based on 16 studies. BMC Cancer 2015, 15, 314. [CrossRef]

13. Zhong, J.-H.; You, X.-M.; Gong, W.-F.; Ma, L.; Zhang, Y.; Mo, Q.-G.; Wu, L.-C.; Xiao, J.; Li, L.-Q. Epidermal Growth Factor Gene Polymorphism and Risk of Hepatocellular Carcinoma: A Meta-Analysis. PLoS ONE 2012, 7, e32159. [CrossRef] [PubMed]

14. Bansal, R.; Van Baarlen, J.; Storm, G.; Prakash, J. The interplay of the Notch signaling in hepatic stellate cells and macrophages determines the fate of liver fibrogenesis. Sci. Rep. 2015, 5, 18272. [CrossRef] [PubMed]

15. Wang, H.; Song, X.; Liao, H.; Wang, P.; Zhang, Y.; Che, L.; Zhang, J.; Zhou, Y.; Cigliano, A.; Ament, C.; et al. Overexpression of Mothers Against Decapentaplegic Homolog 7 Activates the Yes-Associated Protein/NOTCH Cascade and Promotes Liver Carcinogenesis in Mice and Humans. Hepatology 2020, 74, 248-263. [CrossRef] [PubMed]

16. Misiorek, J.O.; Przybyszewska-Podstawka, A.; Kałafut, J.; Paziewska, B.; Rolle, K.; Rivero-Müller, A.; Nees, M. Context Matters: NOTCH Signatures and Pathway in Cancer Progression and Metastasis. Cells 2021, 10, 94. [CrossRef]

17. Ni, M.-M.; Wang, Y.-R.; Wu, W.-W.; Xia, C.-C.; Zhang, Y.-H.; Xu, J.; Xu, T.; Li, J. Novel Insights on Notch signaling pathways in liver fibrosis. Eur. J. Pharmacol. 2018, 826, 66-74. [CrossRef] [PubMed]

18. Villanueva, A.; Alsinet, C.; Yanger, K.; Hoshida, Y.; Zong, Y.; Toffanin, S.; Rodriguez-Carunchio, L.; Solé, M.; Thung, S.; Stanger, B.Z.; et al. Notch Signaling Is Activated in Human Hepatocellular Carcinoma and Induces Tumor Formation in Mice. Gastroenterology 2012, 143, 1660-1669.e7. [CrossRef]

19. Ge, M.; Liu, H.; Zhang, N.; Niu, W.; Lu, Z.; Bao, Y.; Huang, R.; Yu, D.; Shao, R.; He, H. Costunolide represses hepatic fibrosis through WW domain-containing protein 2-mediated Notch3 degradation. Br. J. Pharmacol. 2019, 177, 372-387. [CrossRef] [PubMed]

20. Chen, Y.; Zheng, S.; Qi, D.; Zheng, S.; Guo, J.; Zhang, S.; Weng, Z. Inhibition of Notch Signaling by a $\gamma$-Secretase Inhibitor Attenuates Hepatic Fibrosis in Rats. PLoS ONE 2012, 7, e46512. [CrossRef]

21. Hemati, H.; Kaur, J.; Sobti, R.; Trehanpati, N. Inhibition of NOTCH signaling pathway chemosensitizes HCC CD133+ cells to vincristine and 5-fluorouracil through upregulation of BBC3. Biochem. Biophys. Res. Commun. 2020, 525, 941-947. [CrossRef]

22. Moore, G.; Annett, S.; McClements, L.; Robson, T. Top Notch Targeting Strategies in Cancer: A Detailed Overview of Recent Insights and Current Perspectives. Cells 2020, 9, 1503. [CrossRef]

23. Cao, Z.; Lis, R.; Ginsberg, M.; Chavez, D.; Shido, K.; Rabbany, S.Y.; Fong, G.-H.; Sakmar, T.; Rafii, S.; Ding, B.-S. Targeting of the pulmonary capillary vascular niche promotes lung alveolar repair and ameliorates fibrosis. Nat. Med. 2016, 22, 154-162. [CrossRef]

24. Xu, K.; Moghal, N.; Egan, S.E. Notch signaling in lung development and disease. In Notch Signaling in Embryology and Cancer; Springer: New York, NY, USA, 2012; Volume 727, pp. 89-98. [CrossRef]

25. Yu, T.; Han, C.; Zhu, G.; Liao, X.; Qin, W.; Yang, C.; Liu, Z.; Su, H.; Liu, X.; Yu, L.; et al. Prognostic value of Notch receptors in postsurgical patients with hepatitis B virus-related hepatocellular carcinoma. Cancer Med. 2017, 6, 1587-1600. [CrossRef] [PubMed]

26. Ostojic, A.; Markotic, A.; Kelava, T.; Mrzljak, A. Association between CXCL9/10 polymorphisms and acute rejection of liver allograft. Medicine 2019, 98, e14612. [CrossRef] [PubMed]

27. Storey, J.D. A direct approach to false discovery rates. J. R. Stat. Soc. Ser. B Stat. Methodol. 2002, 64, 479-498. [CrossRef]

28. Huang, Z.; Guo, X.; Zhang, G.; Liang, L.; Nong, B. Correlation between PNPLA3 rs738409 polymorphism and hepatocellular carcinoma: A meta-analysis of 10,330 subjects. Int. J. Biol. Markers 2019, 34, 117-122. [CrossRef] 
29. Chen, X.; Zhou, P.; De, L.; Li, B.; Su, S. The roles of transmembrane 6 superfamily member 2 rs58542926 polymorphism in chronic liver disease: A meta-analysis of 24,147 subjects. Mol. Genet. Genom. Med. 2019, 7, e824. [CrossRef]

30. Hu, B.; Phan, S.H. Notch in fibrosis and as a target of anti-fibrotic therapy. Pharmacol. Res. 2016, 108, 57-64. [CrossRef] [PubMed]

31. Duan, J.-L.; Ruan, B.; Yan, X.-C.; Liang, L.; Song, P.; Yang, Z.-Y.; Liu, Y.; Dou, K.-F.; Han, H.; Wang, L. Endothelial Notch activation reshapes the angiocrine of sinusoidal endothelia to aggravate liver fibrosis and blunt regeneration in mice. Hepatology 2018, 68, 677-690. [CrossRef]

32. Fattovich, G.; Stroffolini, T.; Zagni, I.; Donato, F. Hepatocellular carcinoma in cirrhosis: Incidence and risk factors. Gastroenterology 2004, 127, S35-S50. [CrossRef]

33. Huang, Q.; Li, J.; Zheng, J.; Wei, A. The Carcinogenic Role of the Notch Signaling Pathway in the Development of Hepatocellular Carcinoma. J. Cancer 2019, 10, 1570-1579. [CrossRef]

34. Tanabe, K.K.; Lemoine, A.; Finkelstein, D.M.; Kawasaki, H.; Fujii, T.; Chung, R.T.; Lauwers, G.Y.; Kulu, Y.; Muzikansky, A.; Kuruppu, D.; et al. Epidermal Growth Factor Gene Functional Polymorphism and the Risk of Hepatocellular Carcinoma in Patients With Cirrhosis. JAMA 2008, 299, 53-60. [CrossRef]

35. Zhang, S.; Jiang, J.; Tang, W.; Liu, L. Methylenetetrahydrofolate reductase C677T (Ala > Val, rs1801133 C > T) polymorphism decreases the susceptibility of hepatocellular carcinoma: A meta-analysis involving 12,628 subjects. Biosci. Rep. 2020, 40, BSR20194229. [CrossRef] [PubMed]

36. Baghdadi, I.; Abu Ella, K.; El Shaaraway, A.; Elshayeb, E.; El-Rebey, H.S.; El Hoseeny, M.M.; Naguib, M.; Nada, A. Genetic Polymorphism of Epidermal Growth Factor Gene as a Predictor of Hepatocellular Carcinoma in Hepatitis C Cirrhotic Patients. Asian Pac. J. Cancer Prev. 2020, 21, 2047-2053. [CrossRef] [PubMed]

37. Zhang, S.; Qiao, K.; Trieu, C.; Huo, Z.; Dai, Q.; Du, Y.; Lu, W.; Hou, W. Genetic Polymorphism of Epidermal Growth Factor rs4444903 Influences Susceptibility to HCV-Related Liver Cirrhosis and Hepatocellular Carcinoma in a Chinese Han Population. Clin. Lab. 2017, 63, 845-850. [CrossRef] [PubMed]

38. Pineda-Tenor, D.; Gómez-Moreno, A.Z.; Sánchez-Ruano, J.J.; Artaza-Varasa, T.; Virseda-Berdices, A.; Fernández-Rodríguez, A.; Mendoza, P.M.; Jiménez-Sousa, M.; Resino, S. MTHFR rs1801133 Polymorphism Is Associated With Liver Fibrosis Progression in Chronic Hepatitis C: A Retrospective Study. Front. Med. 2020, 7, 582666. [CrossRef]

39. Khlaiphuengsin, A.; Kiatbumrung, R.; Payungporn, S.; Pinjaroen, N.; Tangkijvanich, P. Association of PNPLA3 Polymorphism with Hepatocellular Carcinoma Development and Prognosis in Viral and Non-Viral Chronic Liver Diseases. Asian Pac. J. Cancer Prev. 2016, 16, 8377-8382. [CrossRef] 